\title{
VLA OBSERVATIONS AT 333 MHZ: A SEARCH FOR PANCAKES
}

\author{
JUAN M. USON, DURGADAS S. BAGRI and T. J. CORNWELL \\ National Radio Astronomy Observatory, P.O. Box. 0, Socorro, NM 87801
}

\begin{abstract}
The P-band is the most recently completed observing band at the VLA. The aperture efficiency of the antennas is 0.4 and the system temperature is $\sim 130 \mathrm{~K}$. The primary beam is slightly off-axis and its half-power width is steeply frequency dependent, which induces abnormal spectral indices on off-axis $\left(\geq 1^{\circ}\right)$ sources. We have searched for Zel'dovich "pancakes" and reached a level of $\Delta \mathrm{S}_{\mathrm{rms}} \sim 3.5 \mathrm{mJy}$ for spectral channels of width $\Delta \nu \sim 100 \mathrm{kHz}$. We find no pancake with $\mathrm{M}_{\mathrm{HI}} \gtrsim 5 \times 10^{14} \mathrm{M}_{\odot}$.
\end{abstract}

\section{THEORETICAL SCENARIO}

Massive, possibly flattened (hence their nickname), protoclusters of galaxies were the first structures to form if the universe evolved along the path described by the so-called "top-down" scenario (Zel'dovich 1970). Their detection would lend strong support to this theory whereas ruling out their existence would exclude the scenario from further consideration. They are expected to contain $\approx 10^{14} \mathrm{M}_{\odot}$ of neutral hydrogen and hence should be detectable through their (redshifted) " $21 \mathrm{~cm}$ " line emission, Doppler-broadened to a half-width in the range $200-700 \mathrm{kHz}$ which should be observable against the featureless galactic background or narrow recombination lines. Their flux depends on the values of key cosmological parameters and should be $\sim$ few mJy (Sunyaev and Zel'dovich 1975). Past attempts to detect them have been unsuccessful.

\section{THE VLA P-BAND SYSTEM}

The VLA P-band system is an afterthought. The feed is a pair of crossed dipoles mounted on the secondary reflector. Spatial constraints preclude accessibility to the prime focus and the effective phase center of the dipole-subreflector system is about one-half wavelenght out-of-focus. This results in a slight broadening of the antenna beam which has a half-power width of about $145^{\prime}$ at $333 \mathrm{MHz}$ and an aperture efficiency of about 0.4. We have found a strong frequency dependence of the half-power beamwidth. For example, between $330 \mathrm{MHz}$ and $335 \mathrm{MHz}$, the change in the half-power beamwidth is about three times that expected of a correctly focused antenna. This induces steep spectral indices $(\alpha \sim 5-10)$ on sources located beyond about $1^{\circ}$ from the center of the beam. In addition, the beam is not centered on the symmetry axis of the antennas but is systematically displaced by about $8^{\prime}$, with a beam-squint of about $3^{\prime}$. We believe that this is 
due to the subreflector, which is asymmetric and shaped as it needs to illuminate the higher frequency Cassegrain off-axis feeds. This induces a phase gradient in the wavefront at $P$-band which results in the small shift that we measure. This effect has been neglected in the past, i. e. the antennas have been slightly but systematically mispointed in (azimuth, elevation) at this band.

Linearly polarized signals from the two dipoles are fed to a low-loss quadrature hybrid network, followed by $30 \mathrm{~dB}$ directional couplers. The receiver is a two-stage GaAs FET room-temperature amplifier. The system temperature is about $130 \mathrm{~K}$, where the receiver noise contribution is $\leq 30 \mathrm{~K}$.

\section{OBSERVATIONS}

We have used the VLA in its most compact configuration. We observed a field centered at $\alpha_{1950}=10^{\mathrm{h}} 50^{\mathrm{m}}, \delta_{1950}=+57^{\circ} 00^{\prime}$ on 1987, March $4,11,13,20,21$ and 25 for a total of 34 hours on source. We observed in spectral mode $1 \mathrm{~A}$ using on-line hanning-smoothing which resulted in 127 contiguous channels of width $24.4 \mathrm{kHz}$ centered at $333.125 \mathrm{MHz}$. Only right-hand circular polarization could be recorded at that time. Phase and bandpass calibration was achieved through interspersed (every $35 \mathrm{~min}$ ) observations of 3C196 and 3C295. We adopt a flux of 60.373 Jy for $3 \mathrm{C} 295$. Standard procedures were used to calibrate the data, which were then examined baseline by baseline in frequency vs. time plots. Baselines obviously contaminated by cross-talk were discarded at this stage $(<20 \%)$.

\section{DATA REDUCTION}

Processing of the Continuum The observations produce a continuum "channel" which is the average of the central $75 \%$ of the observed band. A 1024 by 1024 image with $50^{\prime \prime}$ square-pixels was made and cleaned. This "blank" field has a total flux of $\sim 13 \mathrm{Jy}$. Sources more than $\sim 1^{\circ}$ from the center of the beam show appreciable distortion. A correct image of such a wide field-of-view requires a full three-dimensional Fourier inversion of the visibilities (Cornwell and Perley 1990). We have made a continuum cube of images of size 1024 by 1024 by 16 with $50^{\prime \prime}$ square-pixels which was subsequently cleaned. The projected image again contains a total flux of $\sim 13 \mathrm{Jy}$. However, the sources do no longer show any significant distortion. The field is dominated by point sources.

Processing of the Spectral Data Cleaning the spectral images independently could lead to spurious detections of spectral signals due to the inherent chromaticity of any interferometer. The standard procedure is to evaluate the theoretical visibility that results from the continuum sources in the field at each baseline and frequency; these are then subtracted from the measured visibilities. The residuals are then used to make a "dirty" image for each spectral channel. Their average is then subtracted from all the channel images in order to remove residual continuum emission, which gives the final spectral images. These images showed a distinctive pattern of horizontal stripes. The slow "fringe rate" that results when a baseline is aligned with the direction in which the antennas are pointing allows the baseline to dwell on recording data corresponding to only a few cells in the uv-plane which allows external interference to add up coherently. 
As external interference does not participate in the general rotation of the sky with respect to the array it mimics a source located at the north celestial pole. Therefore, the stripes are actually the far sidelobes of a (strong) source located at the north celestial pole (the south pole is below the horizon). We have found that all images improved significantly when the data taken on and around the vaxis were eliminated. This further reduced the number of visibilities by about $5 \%$ to $7 \%$ but the noise in the images diminished and the artifacts disappeared. The images were then averaged by fours to produce spectral images corresponding to "channels" of width about $100 \mathrm{kHz}$. The rms noise comes within $15 \%$ of the theoretical value as is shown in Fig. 1 (filled symbols, horizontal line). There is a small rise towards the edges of the band which we attribute to a combination of the poorer response of the electronics/correlator at the edges of the band coupled with the systematic errors that we discuss below. The open symbols correspond to using the 2-D continuum image in order to subtract the continuum flux. We attribute the steeper rise in the rms noise toward the edges of the band to the misrepresentation of the continuum sources that follows from using the narrowfield approximation in an inherently wide-field case. Of course, the subtraction of the average spectral image from the individual spectral images favors the central channels which therefore show smaller rms noise values, comparable to those obtained using the 3-D continuum image.

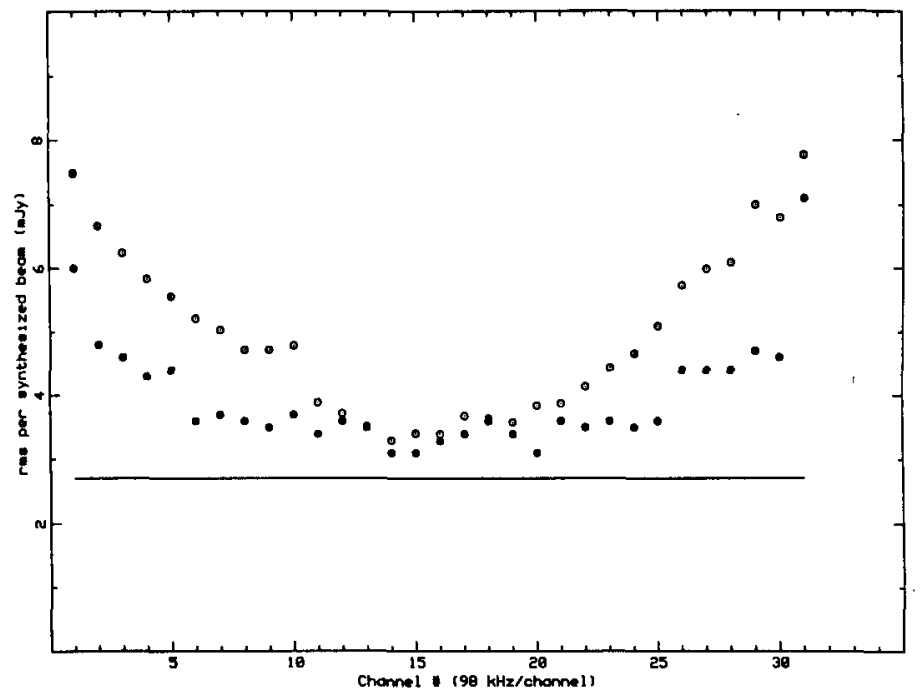

Brief Discussion of Systematic Effects We have found that the flux of sources located further than about $1^{\circ}$ from the center of the beam is modulated as the sky rotates with respect to the antennas. For example, the hourly averages for the flux of the dominant source in the field vary between $0.9 \mathrm{Jy}$ and $1.4 \mathrm{Jy}$. This is consistent with the mispointing of the array elements described above.

Some of the brightest sources in the field leave behind ghosts in the spectral images, appearing as positive (residual) sources at the lower frequencies and negative at the higher end of the band. This effect is due to the steep variation of the beam shape with frequency and again consistent with our measured beam patterns. We have devised a scheme to correct the data for this effect in the future. At this time, we have analyzed our spectral images after excising the 
cells occupied by the strongest ten continuum sources. We have examined these lines-of-sight independently to ensure that no "pancake" is missed by excluding them from the analysis described in the next section.

\section{ANALYSIS AND DISCUSSION}

We have analyzed our cube of images as follows. We used spectral planes 4 through 28 in order to avoid the increase in noise that appears at the band edges. We have used several matched linear filters to examine the images (Gaussian profiles with half-width between $300 \mathrm{kHz}$ and $700 \mathrm{kHz}$ ). The distributions of amplitudes are gaussian with no statistically significant outliers. Proper care was taken to evaluate in each case the effective number of degrees of freedom (independent samples) in our cube. Therefore, we have detected no pancakes. We place lower limits on the mass of any pancake in our field that correspond to twice the maximum deviation in the amplitude distributions $(6 \sigma)$. Indeed, although our noise is gaussian-distributed, the "signal" is not -at most there could be one pancake in our field; which excludes the statistical methods that are normally used to set upper limits from noise-limited data. Any "pancake" with higher flux would have produced in any case a signal stronger that our highest noise bump, and is therefore excluded by our measurement. We have taken into account that our average efficiency inside the half-peak response of the primary beam is 0.72 and corrected our limits accordingly. We would like to emphasize that these kinds of measurements cannot be analyzed in the usual statistical way, although this has unfortunately been common practice. Therefore, published limits should be carefully reevaluated.

The implications of these upper limits on the masses of the fiducial pancakes are strongly dependent on the cosmological model adopted. We exclude (HI) masses that range from $7 \times 10^{13} \mathrm{~h}^{-2} \mathrm{M}_{\odot}$ for the case of a pancake with line of sight rms velocity dispersion of $300 \mathrm{~km} \mathrm{~s}^{-1}$ in a cosmologically flat (EinsteindeSitter) universe to $3 \times 10^{14} \mathrm{~h}^{-2} \mathrm{M}_{\odot}$ if the structure is not collapsed $(\Delta \mathrm{v}=700$ $\mathrm{km} \mathrm{s}^{-1}$ ) and the universe is open ( $\mathrm{h}$ is Hubble's constant in units of $100 \mathrm{~km} \mathrm{~s}^{-1}$ $\mathrm{Mpc}^{-1}$ ). These limits do not seriously constrain the Zel'dovich scenario as our surveyed volume does not necessarily contain a "pancake". We are proceeding with further observations that will eventually cover a sufficiently large volume to ensure having a pancake in our sample if the scenario is correct.

\section{ACKNOWLEDGEMENTS}

We have benefitted from many helpful discussions with Barry Clark and Peter Napier on all experimental details of this project. We thank Anantharamaiah, Jacqueline Van Gorkom and Steve Van Hook for help with part of this work.

Cornwell, T. J. and Perley, R. A. (1990), in "Digital Image Synthesis and Inverse Optics", Arthur F. Gmitro, Paul S. Idell, Ivan J. LaHaie, Eds., Proc. SPIE $1351, \mathrm{p} 706$.

Sunyaev, R. A. \& Zel'dovich, Ya. B. 1975, M.N.R.A.S., 171, 375.

Zel'dovich, Ya. B. 1970, Astr. Ap., 5, 84. 
Namir Kassim: Juan, I was recently on an observing run at the Arecibo telescope. We were searching for low frequency recombination lines for which a $200 \mathrm{MHz}$ feed was built. I've heard that Bernie Burke is planning to use this system to look for pancakes. Since, as you pointed out earlier, this lower frequency is where you would have liked to look for red-shifted pancakes had you not been restricted to the available P-band VLA system, would you say this experiment has a better chance of success?

Juan Uson: I do not know the constraints and especially the sources of systematic errors and interference at Arecibo at $220 \mathrm{MHz}$. The experiment is certainly worth trying, but I cannot comment on its chances of success.

L. Gurvits: You have shown us a very interesting technique which could be applied to many problems. But would you please repeat the astrophysical reasons for the reported work. It seems that it is a rather well accepted fact that there must not be any pancakes at such a late epoch as $z=3.3$. Juan Uson: We have observed at $333 \mathrm{MHz}$ because that is where the VLA receivers work. The evolution of pancakes might result in significant fragmentation into smaller clumps by $z=3.3$ but this must not be a problem as our beam would not resolve them out; their signal is not lost unless the fragmentation results in the ionization of the atomic hydrogen. Besides, although current theories suggest we should probe higher redshifts, the scenarios would easily accommodate a detection at $z=3.3$ !

John Baldwin: The bandwidths you quote of 300 and $600 \mathrm{kHz}$ for pancake $\mathrm{HI}$ limits seems quite small. Is there any difficulty in analysing the data for larger bandwidths.

Juan Uson: Our total bandwidth is $\sim 3 \mathrm{MHz}$. We could analyze the cubes of images looking for signals with somewhat larger widths although the widths used correspond to the line-of-sight velocity dispersions of about $300-600$ $\mathrm{km} / \mathrm{sec}$ which seem adequate. 